\title{
The Use of Osteopathic Manipulative Medicine in the Management of Recurrent Mastitis
}

Caitlin Jackson, OMS III; Brian Loveless, DO

From the Western University of Health Sciences, College of Osteopathic Medicine of the Pacific in Pomona, California.

Financial Disclosures: None reported.

Support: None reported.

Address correspondence to

Brian Loveless, DO, Department of NMM/OMM,

Western University of Health

Sciences, College of Osteopathic Medicine of the Pacific, 309 E. Second Street, Pomona, CA 91766-1854.

Email:

bloveless@westernu.edu

Submitted

March 4, 2020;

revision received

July 17, 2020;

accepted

August 4, 2020.
Mastitis affects breastfeeding mothers everywhere and management obstacles often lead to cessation of breastfeeding. Breastfeeding mastitis is commonly managed with antibiotics despite lack of clear infectious etiology. With the emerging problem of antibiotic resistance, novel managements are required. We present the case of a 34-year-old woman with 6 pregnancies and 3 children (gravida 6, parity 3) who had 5 cases of mastitis within 6 months treated with multiple courses of antibiotics. The patient underwent with osteopathic manipulative treatment (OMT) to the affected breast over 2 sessions and was taught how to perform self-myofascial release. Techniques are shown in an accompanying Supplemental Video. As of this report, the patient had been symptom free for 1 year. Future research, including a clinical trial of OMT, is required to determine whether osteopathic physicians can effectively manage recurrent lactational mastitis.

J Am Osteopath Assoc. 2020;120(12):921-925. Published online October 27, 2020. doi:10.7556/jaoa.2020.143

Keywords: breastfeeding, lactation, mastitis, OMT, osteopathic manipulative treatment

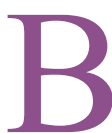

reastfeeding is widely accepted as beneficial to both mother and infant. ${ }^{1}$ However, complications such as breast engorgement and mastitis can be an obstacle to breastfeeding. Mastitis presents as inflammation of the breast tissue, with or without infection, and is most associated with lactation. The diagnosis of mastitis requires an erythematous and painful area of the breast and constitutional symptoms such as fatigue, myalgias, fever, and chills. Mothers sometimes consider breastfeeding cessation to relieve their pain, despite it not being recommended; cessation may exacerbate the condition. ${ }^{2}$ The incidence of mastitis is not well defined, ranging from $2.5 \%$ to $33 \%$ depending on the population and study, with the single greatest risk factor being a history of previous mastitis. ${ }^{3,4}$ The cause of mastitis is unclear, with milk stasis, infectious pathogens, stress, and nipple trauma considered as possible etiologies. Current management recommendations for mastitis include supportive measures, such as analgesia and warm compresses. Antibiotics may be indicated, depending on the duration and severity of infection. ${ }^{4,5}$ In this case report, we describe a woman with recurrent mastitis that was successfully managed with osteopathic manipulative treatment (OMT). A literature review revealed no other cases of recurrent mastitis treated with OMT.

\section{Report of Case}

A 34-year-old woman with 6 pregnancies and 3 children (gravida 6 parity 3; G6P3) was referred to our clinic with a complaint of recurrent left breast infections for the past 6 
months. When she presented, she was in the midst of her $5^{\text {th }}$ episode of lactational mastitis in the left breast, which had been treated with multiple courses of antibiotics. She was first diagnosed 21 weeks postpartum with left breast mastitis at an urgent care clinic, where she was started on $500 \mathrm{mg}$ of amoxicillin 3 times per day, later changed to $500 \mathrm{mg}$ of cephalexin twice per day. Her symptoms resolved, but 4 to 5 days after completion of her antibiotic course, redness and pain returned. She was seen by a primary care physician in our clinic, and again treated with $500 \mathrm{mg}$ of cephalexin 3 times per day for 10 days.

The patient returned to her primary care office at approximately 24 weeks postpartum with redness and tenderness in her left breast, along with fever and chills, for the prior 2 days. At that time, a culture was taken of her milk, and she was prescribed trimethoprim/sulfamethoxazole (TMP/SMX) twice per day for 10 days. The next day, she developed a rash, and the TMP/SMX prescription was changed to dicloxacillin. Her milk culture grew group A streptococcus. No changes were made at that time.

About 7 weeks later, at 31 weeks postpartum, the patient again developed pain and redness in her left breast and chills, and she returned to her primary care office. At that time, she was only pumping milk from her left breast because of worries about the baby being infected, and she was considering weaning her 7month-old baby from breastfeeding altogether. She was given a 1-gram intramuscular dose of ceftriaxone and prescribed 875/125 mg amoxicillin/potassium clavulanate twice per day for 10 days. She was also referred to the infectious disease department for further evaluation. The patient saw an infectious disease consultant 4 days later; she was relatively asymptomatic, and no changes to her course of treatment were recommended at that time. Again, the patient stated that she was willing to wean if it would prevent recurrence of her infections. At 36 weeks postpartum, she again developed symptoms, including myalgias, and she was given another course of amoxicillin/potassium clavulanate.
She scheduled an appointment in the osteopathic manipulative medicine (OMM) clinic on the recommendation of a friend, and she was seen 5 days after her most recent episode. She had completed half of her course of antibiotics, and she had only mild symptoms of pain and redness at the time of her evaluation. She was no longer experiencing myalgias. She had stopped breastfeeding but was pumping approximately 1 ounce of milk per breast at a time. She reported no problems with mastitis with previous children, and she was able to breastfeed them successfully.

On physical examination by an author (B.L.) with a chaperone present, the patient had some tenderness of the medial left breast with fascial restrictions palpable in the medial lower quadrant. There was no redness or fluctuant mass noted, and the nipple appeared normal. A detailed structural examination revealed restriction of the left thoracic inlet with increased fullness of the left supraclavicular fossa and tension palpable in Sibson's fascia on the left. The left pectoral and axillary fascia were restricted, and the left thoracic diaphragm showed decreased motion with inhalation. Following examination, the physician (B.L.) performed OMT to the thoracic and abdominal regions. Specific techniques included myofascial release to the breast, pectoral fascia, and thoracic diaphragm, balanced ligamentous techniques to the thoracic inlet, and thoracic lymphatic pump technique (Supplemental Video). The patient's antibiotics were discontinued, as they were not felt to be indicated at that time. She was shown how to do selfmyofascial release for her breasts, particularly stretches of the soft tissue in the cardinal planes.

One week later, at approximately 38 weeks postpartum, she returned to the OMM clinic, noting that she had developed some tenderness in the left breast the night before. Her symptoms were mild at that time, with some aching and mild redness of the left breast. On physical examination with a chaperone present, fascial restrictions were again noted in the medial lower quadrant of the left breast. The lobules were firm and swollen, but no masses or abscess were noted. The patient had a restriction of the left thoracic inlet, left 
thoracic diaphragm, left pectoral fascia, and left scapula. OMT was again performed, including myofascial release to the above dysfunctions and a thoracic lymphatic pump.

After discussing options with the patient, a decision was made to watch and wait. The patient was advised to contact her primary care provider in the case of worsening symptoms, fever, or other constitutional symptoms to restart antibiotics. The patient contacted the physician (B.L.) about 6 months after her second visit to report that she had no infection since the last visit. She reported sufficient supply, had resumed breastfeeding on demand, and she and her baby were doing well. The patient was contacted at 1 year after her first visit and she reported no further episodes of mastitis; she had continued successful breastfeeding of her now 19-month-old son.

\section{Discussion}

Current mastitis management guidelines from both the World Health Organization ${ }^{4}$ and the Academy of Breastfeeding Medicine $^{2}$ recommend conservative methods before antibiotics, including effective milk removal, dietary changes, heat or ice, and oral analgesics. However, Foxman et $\mathrm{al}^{3}$ concluded that conservative managements for mastitis were minimally effective on their own, and most cases of mastitis are eventually treated with antibiotics. ${ }^{2,4}$ Antibiotics are often effective at eradicating the infection, but recurrence is not uncommon. The actual recurrence rate is not clearly defined in the literature.

The osteopathic approach to patient care attempts to address as many contributing factors in a patient's illness as possible, taking a health-focused view of the patient. The causes of a patient's presentation can be viewed through 5 models: biomechanical, respiratorycirculatory, neurologic, metabolic, and biopsychosocial. ${ }^{6}$ These 5 models were developed by the Educational Council of Osteopathic Principles (ECOP) of the American Association of Colleges of Osteopathic Medicine (AACOM) ${ }^{7}$ as a framework to ensure a well-rounded plan of care. The biomechanical model addresses factors to do with posture and body movement, including but not limited to joints, muscle tonicity, and fascia. For this case of mastitis, with this model in mind, we treated the fascia and soft tissues of the breast and related lymphatic structures. The respiratory-circulatory model addresses fluid flow throughout the body, including arterial flow, venous return, lymph, and oxygen. This case required management via this model to improve lymphatic drainage of the breast, along with pump treatments to the thoracic region to increase lymphatic return to the heart. The neurologic model addresses the autonomic system, pain perception, and viscerosomatic and somatovisceral reflexes. The reduction of pain in this patient from the manual treatment addresses the neurologic model, whereby reducing nociception seems to reduce sympathetic activity, thereby promoting an effective immune response. The metabolic model was addressed in this case by discussing an improved diet, heat or ice locally applied, and the addition of oral analgesics when indicated. Finally, the biopsychosocial model addresses the psychological impact of a diagnosis or condition on the patient.

In this case, we provided reassurance regarding the self-limiting nature of the presentation, suggested behavioral changes such as increased feeding on the affected breast, and promoted continued breastfeeding, as re-establishment of successful breastfeeding can be a coping mechanism to improve stress and anxiety in patients. ${ }^{8}$ Lastly, we also taught the patient some selftreatment techniques for her breast, consisting of taking the breast tissue in the cardinal directions as a stretch. This last step was done to empower the patient in light of what had been a long and emotionally draining condition, further supporting the patient from the biopsychosocial model perspective.

Breast massage has been documented in the literature for a variety of conditions, including plugged ducts, mammary gland hyperplasia, milk stasis, engorgement, and mastitis. Most studies show improvement in patient presentations. ${ }^{9-12}$ Many of the manual techniques 
studied in the previous literature overlap with the techniques used in this case, such as myofascial release, soft tissue, and articulatory techniques. No studies have documented the use of OMT specifically. The mastitis protocol from the Academy of Breastfeeding Medicine $^{2}$ specifically calls for research on breast massage. As the incidence of mastitis with MRSA and other serious infections such as breast abscess increases, effective managements that do not require antibiotics are urgently needed. ${ }^{13}$ Additionally, there is a paucity of research on recurrent mastitis in the literature. One study ${ }^{5}$ proposed recurrent mastitis as resulting from shortened courses of antibiotics, or the presence of Staphylococcus carriers. Another study ${ }^{14}$ found that the presence of infectious bacterial strains are not correlated with infectious lactational mastitis. This lack of definition highlights the difficulty of adequately assessing and managing mastitis and recurrent mastitis. We agree that in many cases, including ours, the etiology is unclear. Taking a patient-centered approach such as the framework offered by osteopathic medicine, including investigating somatic causes for mastitis, increases the chance of an appropriate management plan being formulated. Further research into defining mastitis and the causes of these cases would aid in management.

\section{Conclusion}

Lactational mastitis is a common presentation in many primary care settings, but the true cost of care has not been adequately studied. This case of recurrent mastitis previously managed with multiple courses of antibiotics was resolved after 2 treatments of OMT. Given global concerns with antibiotic resistance and the high prevalence of mastitis, this easy-to-administer, accessible, low-cost, and noninvasive therapy should be considered as a promising alternative. Although osteopathically-trained physicians are found in many settings where patients with mastitis present, similar techniques could also be taught to other providers and to lactation consultants caring for breastfeeding women. Moreover, it might be considered not only in cases of recurrent mastitis that have not been responsive to antibiotics but also as an addition to conservative care during initial presentation. Further research is needed to examine efficacy, determine best practices, and potentially develop a more standardized protocol for management.

\section{Author Contributions}

Both authors provided substantial contributions to conception and design, acquisition of data, or analysis and interpretation of data; both authors drafted the article or revised it critically for important intellectual content; both authors gave final approval of the version of the article to be published; and both authors agree to be accountable for all aspects of the work in ensuring that questions related to the accuracy or integrity of any part of the work are appropriately investigated and resolved.

\section{References}

1. Section on Breastfeeding. Breastfeeding and the use of human milk. Pediatrics. 2012;129(3):e827-e841. doi:10.1542/peds.2011-3552

2. Amir LH, Academy of Breastfeeding Medicine Protocol Committee. ABM clinical protocol \#4: Mastitis, revised March 2014. Breastfeed Med. 2014;9(5):239-243. doi:10.1089/bfm.2014.9984

3. Foxman B, D'Arcy H, Gillespie B, Bobo JK, Schwartz K. Lactation mastitis: occurrence and medical management among 946 breastfeeding women in the United States. Am J Epidemiol. 2002;155 (2):103-114. doi:10.1093/aje/155.2.103

4. World Health Organization. Mastitis: Causes and Management. World Health Organization website. Published 2000. Accessed October 14, 2019. https://www.who.int/maternal_child_adolescent/documents/fch cah_00_13/en/.

5. Boakes E, Woods A, Johnson N, Kadoglou N. Breast infection: a review of diagnosis and management practices. Eur $\mathrm{J}$ Breast Health 2018;14(3):136-143. doi:10.5152/ejbh.2018.3871

6. Hruby RJ, Tozzi P, Lunghi C, Fusco G. The Five Osteopathic Models: Rationale, Application, Integration - From an evidence-based to a person-centered osteopathy. 1st ed. Handspring Publishing Limited; 2015

7. Giusti R. ed. Glossary of Osteopathic Terminology. 3rd ed. American Association of Colleges of Osteopathic Medicine, 2017.

8. Dozier AM, Nelson A, Brownell E. the relationship between life stress and breastfeeding outcomes among low-income mothers. Adv Prev Med. 2012. doi:10.1155/2012/902487

9. Lv $\mathrm{P}$, Chong $\mathrm{Y}$, Zou $\mathrm{H}$, Chen $\mathrm{X}$. A study of using massage therapy accompanied with stretching exercise for rehabilitation of mammary gland hyperplasia. Biomed Res Int. 2016. doi:10.1155/2016/ 9426167

10. Zhao C, Tang R, Wang J, et al. Six-step recanalization manual therapy: a novel method for treating plugged ducts in lactating women. J Hum Lact. 2014;30(3):324-330. doi:10.1177/0890334414532314 
11. Bolman M, Saju L, Oganesyan K, Kondrashova T, Witt AM. Recapturing the art of therapeutic breast massage during breastfeeding. J Hum Lact. 2013;29(3):328-331. doi:10.1177/ 0890334413475527

12. Witt $A M$, Bolman M, Kredit S, Vanic A. Therapeutic breast massage in lactation for the management of engorgement, plugged ducts, and mastitis. J Hum Lact. 2016;32(1):123-131. doi:10.1177/ 0890334415619439
13. Branch-Elliman W, Golen TH, Gold HS, Yassa DS, Baldini LM, Wright SB. Risk factors for staphylococcus aureus postpartum breast abscess. Clin Infect Dis. 2012;54(1):71-77. doi:10.1093/cid/cir751

14. Kvist LJ, Larsson BW, Hall-Lord ML, Steen A, Schalén C. The role of bacteria in lactational mastitis and some considerations of the use of antibiotic treatment. Int Breastfeed J. 2008;3:6. doi:10.1186/ $1746-4358-3-6$

() 2020 American Osteopathic Association 